\title{
Outbreak
}

\section{Molecular diagnosis of echovirus 30 as the etiological agent in an outbreak of aseptic meningitis in Panama: May - June 2008}

\author{
Alexander A. Martinez ${ }^{1,2,3}$, Juan Castillo ${ }^{1}$, Mirla C. Sanchez ${ }^{1}$, Yamitzel Zaldívar ${ }^{1}$, Yaxelis Mendoza $^{1}$, \\ Maribel Tribaldos ${ }^{1}$, Pablo Acosta ${ }^{4}$, Rebecca E. Smith ${ }^{1}$, Juan Miguel Pascale ${ }^{1,5}$ \\ ${ }^{1}$ Gorgas Memorial Institute for Health Studies, Panama City, Panama \\ ${ }^{2}$ Department of Biotechnology, Acharya Nagarjuna University, Guntur - 522 510, A.P. India \\ 3 INDICASAT-AIP Clayton, City of Knowledge 0843-01103, Panama City, Panama \\ ${ }^{4}$ Departament of Epidemiology Ministry of Health, Chiriqui, Panama \\ ${ }^{5}$ Departament of Microbiology, School of Medicine, University of Panama, Panama City, Panama
}

\begin{abstract}
Introduction: Aseptic meningitis outbreaks are commonly caused by viral pathogens with enterovirus a common etiological agent. Between May and June of 2008, an outbreak of 173 cases of aseptic meningitis occurred in the Chiriqui Province of Panama. Molecular techniques were used to identify the etiological agent.

Methodology: Cerebrospinal fluid (CSF) samples from 75 patients were received at the Gorgas Memorial Institute for Health Studies. RNA extraction and one-step RT-PCR were performed on each sample to determine the presence of enterovirus. Thirty-four samples which were positive for enterovirus were subject to group-specific PCR, sequencing, and phylogenetic analysis to identify the etiological agent of the outbreak.

Results: The CSF of 58 subjects was found positive for the enterovirus family using RT-PCR. Thirty-four samples were found to belong to the enterovirus B group. Phylogenetic analysis of four successfully sequenced samples revealed echovirus 30 as the etiological agent. Conclusion: Echovirus 30 is reported as the likely cause of an outbreak of aseptic meningitis in Panama, the first since the 1980s.
\end{abstract}

Key words: meningitis, aseptic; enterovirus B, human; echovirus infections; Panama; disease outbreaks; VP1

J Infect Dev Ctries 2012; 6(12):836-841.

(Received 02 March 2012 - Accepted 10 October 2012)

Copyright $($ C 2012 Martinez et al. This is an open-access article distributed under the Creative Commons Attribution License, which permits unrestricted use, distribution, and reproduction in any medium, provided the original work is properly cited.

\section{Introduction}

The human enteroviruses belong to the Picornaviridae family. They are non-enveloped, single-stranded RNA viruses comprising 68 immunologically- and genetically-distinct viruses, classified into four genetic groups, A to D [1]. Transmission of these viruses generally occurs by the respiratory and fecal-oral routes with respiratory transmission predominant in regions of improved hygiene [2]. Infection through contaminated swimming water is also reported [3] and symptoms may be exacerbated by intensive exercise [4]. Epithelial cells of the gastrointestinal and respiratory tracts are primary sites of replication; viremia may follow with infection of secondary organs [2].

Many enterovirus infections are asymptomatic or self-limiting with a non-specific febrile illness or common cold-like symptoms of the upper respiratory tract. Infection may, however, result in more severe illnesses such as hemorrhagic conjunctivitis or acute flaccid paralysis. These viruses predominate in children aged five years or older, individuals with lower socioeconomic status, and males under 20 years of age (2). In recent years, echovirus 30 has been a frequently identified etiological agent in outbreaks of aseptic meningitis throughout the world (5-9).

In 2008, an increase in cases of aseptic meningitis was reported from various regions of Panama, the majority (173 cases) from the province of Chiriquí. This is the second highest record of aseptic meningitis cases in Chiriquí Province since 2002, when 261 cases were reported (Pablo Acosta, personal communication). In the intervening years and in 2001, fewer than 55 cases per year were reported, with an average of 25 cases per year for the years 2001, 20032007 (Pablo Acosta, personal communication). Our study aimed to report the causative agent of the aseptic meningitis outbreak in 2008. 


\section{Methodology}

Sample collection

Between May and June of 2008 in the Chiriquí Province of Panama, 173 patients showed clinical signs and symptoms indicative of aseptic meningitis. All patients presented to the José Domingo de Obaldía Hospital in David, the capital city of Chiriquí, and cerebrospinal fluid (CSF) samples were collected from 75 patients, representing over $40 \%$ of the 173 cases. In this molecular epidemiological study, the CSF samples were subject to chemical and microbiological analyses in David and molecular analysis at the Instituto Conmemorativo Gorgas de Estudios en Salud (ICGES) in Panama City.

\section{Molecular analysis}

Molecular analysis was performed by extracting RNA directly from CSF using the QIAamp Viral RNA Mini Kit (Qiagen, Gaithersburg MD, USA), according to the manufacturer's recommendations. Extracted RNA was subjected to RT-PCR directed at the conserved region in the 5, UTR among the enteroviruses using primers EV1/EV2 for the first PCR and EVD2/EVU2 for the nested PCR, as previously described [10]. Samples showing a $197 \mathrm{bp}$ band by agarose gel electrophoresis were considered positive for enterovirus. To identify the viral species, a hemi-nested PCR was performed using primers to the VP1 region of the enterovirus genome, as previously described [10], and products were visualized on a $1.5 \%$ agarose gel. To identify the specific enterovirus responsible for the meningitis outbreak, we sequenced PCR amplicons with the same primers used in the hemi-nested PCR [10].

\section{Case definitions}

Level I patients were defined as confirmed cases, displaying both clinical signs and microbiological evidence of aseptic meningitis. This group included 58 (of 75) cases of samples positive for enterovirus by PCR, 34 (of 58) positive specifically to enterovirus B by PCR, and 4 (of 34) samples sequenced.

Level II patients were defined as probable cases, displaying only clinical signs of aseptic meningitis. This group included 17 (of 75 received at Gorgas Memorial Institute) samples testing negative for enterovirus by PCR, and 98 (of 173) samples from which CSF samples were not collected at José Domingo de Obaldía Hospital in David.

\section{Phylogenetic analysis}

We performed two phylogenetic analyses. The first analyzed the type of enterovirus involved in the outbreak and the second classified it in the respective lineage. For each analysis, multiple sequence alignments using the samples sequenced in this study and reference strains were performed using CLUSTAL $\mathrm{W}$ and Molecular Evolutionary Genetics Analysis Version 4.0 (MEGA 4) software (MEGA, Tempe, AZ, USA). The maximum composite likelihood method was used to evaluate genetic distances. MEGA 4 software was used for pairwise comparisons and to construct neighbor-joining (NJ) trees. The reliability of the NJ tree was estimated by bootstrap analysis with 1,000 pseudoreplicates.

\section{Results}

Epidemiological and laboratory results

Between 5 May 2008 and 6 June 2008, a season noted for increasing rainfall and humidity in Panama, an increase in meningitis cases among children was observed, with 173 cases reported. All cases were concentrated in Chiriquí Province, which is located in the north-west of Panama (Figure 1). The total population of this province is 368,790 with 118,504 children younger than 14 years old. We received samples from 75 patients of the total group $(n=173)$ who showed clinical evidence of aseptic meningitis and whose CSF showed no bacterial growth after 72 hours' culture in enrichment media. Ages of the affected patients ranged between 6 months to 13 years and $57 \%$ of the patients were older than five years. Glucose concentration in CSF was within normal range for all patients, suggesting a non-bacterial cause of the meningitis. Also, the differential white blood cell counts of CSF showed elevated neutrophils and leukocytes, indicating an infectious cause of the meningitis (Table). A slightly higher incidence of nuchal rigidity in boys was observed, as well as higher leukocyte counts in the CSF in boys under the age of five compared to girls of the same age.

\section{Molecular biology results}

In total, 58 of 75 samples (77\%) were positive for enterovirus. Viral species were determined for 34 of these 58 enterovirus-positive samples (59\%), and all were found to belong to the human enterovirus B species. Four specimens were sequenced and data was submitted to NCBI (accession numbers HM854943HM854946). A phylogenetic analysis showed that the 
Figure 1. Geographic location of an outbreak of aseptic meningitis in Panama, 2008

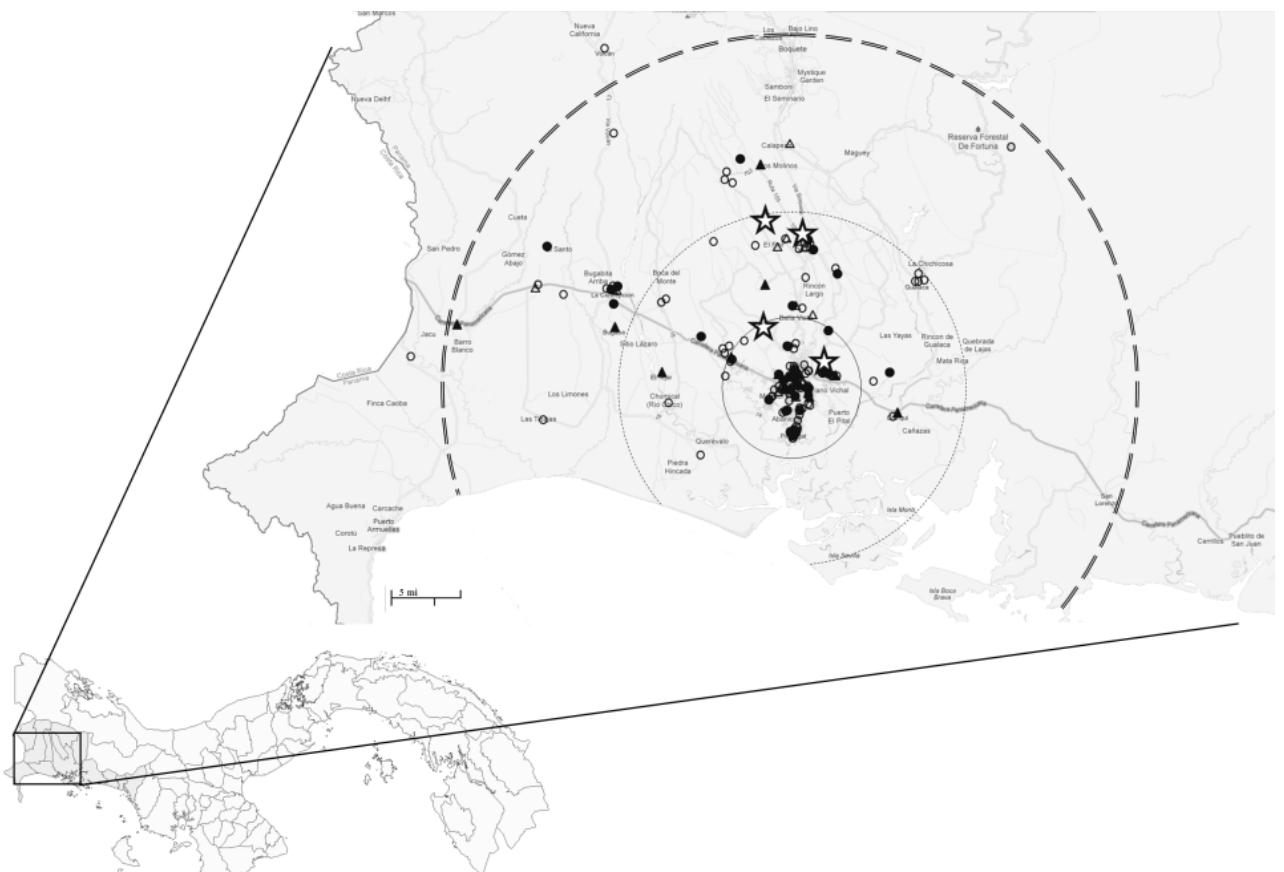

Chiriquí Province is highlighted in the inset and by darker shading on the main map. Stars: sequenced samples; open circles: samples NOT received by the ICGES laboratory; open triangles: samples negative for any type of Enterovirus by PCR; solid triangles: samples positive to Enterovirus by PCR; solid circles: Enterovirus B positive by PCR.

Table. Demographic and clinical data for the 58 patients positive for Enterovirus by molecular methods

\begin{tabular}{|c|c|c|c|c|c|c|c|c|c|c|}
\hline Age & Gender & $\mathbf{n}$ & $\begin{array}{c}\text { Headache } \\
\text { ("yes") } \\
\text { n (\%) }\end{array}$ & $\begin{array}{c}\text { Vomiting } \\
\text { ("yes") } \\
\text { n (\%) } \\
\end{array}$ & $\begin{array}{c}\text { Fever } \\
\text { ("yes") } \\
\text { n (\%) }\end{array}$ & $\begin{array}{l}\text { Nuchal } \\
\text { rigidity } \\
\text { ("yes") } \\
\text { n (\%) } \\
\end{array}$ & $\begin{array}{c}\begin{array}{c}\text { Average } \\
\text { leukocyte } \\
\text { count } \\
\left(\text { cells } / \mathbf{m m}^{3}\right)\end{array} \\
\end{array}$ & $\begin{array}{c}\text { Whole blood } \\
\text { differential count } \\
\text { (\% neutrophils)** }\end{array}$ & $\begin{array}{c}\text { Average } \\
\text { glucose in } \\
\text { CSF } \\
(\mathbf{m g} / \mathbf{d L}) \\
\end{array}$ & $\begin{array}{c}\text { Average } \\
\text { leukocytes in } \\
\text { CSF } \\
\left(\text { cells } / \mathbf{m m}^{3}\right) \\
\end{array}$ \\
\hline \multirow[t]{2}{*}{$0-2$} & $\mathrm{M}$ & 2 & $1(50)$ & $2(100)$ & $2(100)$ & $1(50)$ & 15900 & 76 & 60 & 95 \\
\hline & $\mathrm{F}$ & 2 & $1(50)$ & $2(100)$ & $2(100)$ & $1(50)$ & 16900 & 83 & 54 & 42 \\
\hline \multirow[t]{2}{*}{$3-5$} & $\mathrm{M}$ & 14 & $14(100)$ & $14(100)$ & $14(100)$ & $4(29)$ & 12979 & 87 & 62 & 79 \\
\hline & $\mathrm{F}$ & 7 & $7(100)$ & $7(100)$ & $7(100)$ & $1(14)$ & 11128 & 76 & 64 & 35 \\
\hline \multirow[t]{2}{*}{$0-5$} & $\bar{M}$ & 16 & $\begin{array}{l}15(94) \\
\end{array}$ & 16(100) & $16(100)$ & $\begin{array}{l}5(31) \\
\end{array}$ & 13344 & 86 & $\overline{62}$ & 81 \\
\hline & $\mathrm{F}$ & 9 & $8(89)$ & $9(100)$ & $9(100)$ & $2(22)$ & 12411 & 77 & 62 & 37 \\
\hline \multirow[t]{2}{*}{ 6-10 } & $\overline{\mathrm{M}}$ & 17 & $\overline{~ 17(100)}$ & $16(94)$ & $17(100)$ & 4(24) & 16188 & 87 & 68 & $\overline{56}$ \\
\hline & $\mathrm{F}$ & 12 & $12(100)$ & $11(92)$ & $11(92)$ & $2(17)$ & 14875 & 83 & 60 & 70 \\
\hline $10+*$ & $\mathrm{M}$ & 4 & $3(75)$ & $3(75)$ & $4(100)$ & $3(75)$ & 12050 & 87 & 68 & 111 \\
\hline \multirow{2}{*}{ 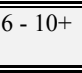 } & $\overline{\mathrm{M}}$ & 21 & $20(95)$ & $19(91)$ & $21(100)$ & 7 (33) & 15400 & 87 & 60 & 66 \\
\hline & $\mathrm{F}$ & 12 & $12(100)$ & $11(92)$ & $11(92)$ & $2(17)$ & 14875 & 83 & 68 & 70 \\
\hline & Total male & 37 & $35(95)$ & $35(95)$ & 37 (100) & $12(32)$ & 14511 & 86 & 66 & 73 \\
\hline & Total female & 21 & $20(95)$ & $20(95)$ & $20(95)$ & $4(19)$ & 13819 & 81 & 56 & 56 \\
\hline & Total & $\overline{258}$ & $55(95)$ & $55(95)$ & $57(98)$ & $16(28)$ & 14260 & 84 & 64 & 67 \\
\hline
\end{tabular}

CSF: cerebrospinal fluid

* No female patients observed in this age rang

** $100 \times$ [Number of neutrophils/(Number of neutrophils + number of lymphocytes)] 
four sequences belonged to the echovirus 30 group of the human enterovirus B species. Analysis of the echovirus 30 lineages showed that the Panamanian viruses were most similar to viruses isolated in Finland and France in 1996 and France in 2005, designated to the F lineage by the nomenclature of Bailly et al. [11] (Figure 2).

\section{Discussion}

The purpose of this epidemiologic study was to identify the causative agent of the outbreak of aseptic meningitis in a specific geographic region of Panama. Our data indicate that human enterovirus B species was isolated in at least $30 \%$ of the cases (Level I, confirmed) of the outbreak observed in Chiriquí during May and June 2008, and more specifically that echovirus 30 was implicated in at least four cases. The remaining cases, classified as Level II, showed only clinical signs of aseptic meningitis and we cannot wholly discount other infectious agents being present and the cause of illness in these patients, or in the patients for which CSF tested negative for enterovirus. As reported in other enterovirus outbreaks involving patients aged under 20 years [12,13], male patients were observed more frequently than female patients (1.8:1), although concurrent echovirus 30 outbreaks in Argentina (2007-2008) and Korea (2008) showed a gender ratio of $1: 1$ and $1.31: 1$, respectively. The way by which echovirus 30 spread through the community of Chiriquí is unknown, but $50 \%$ of the patients were older than 6 years, implicating common environments, such as schools. Panama's cohort of patients were of a similar age compared to those reported in Argentina, where a mean age of 4.3 years was observed [7]. In Korea, a median age of 5.8 years was observed for Echovirus 30 patients [8].

The enterovirus B echovirus 30 outbreak observed in Panama during 2008 was not unique to this region. Several other countries reported outbreaks with echovirus 30 during 2008, including Korea (May to October 2008) [8]; Japan (September 2008) [4] and Argentina (2007 - 2008) [7]. One goal of our study was to compare clinical data for the Panamanian outbreak with those from other outbreaks that occurred in 2008. In Panama, nearly $100 \%$ of cases presented with headache, vomiting and fever, similar to the outbreaks reported in the outbreaks in Japan and at other sites [4]. However, in Panama, only $28 \%$ of patients showed neck stiffness, while in Japan and other locations, at least $60 \%$ of patients reported this sign [4]. Hayashi et al. [4] note that even in the
Figure 2. Phylogenetic tree with Panamanian samples and reference strains

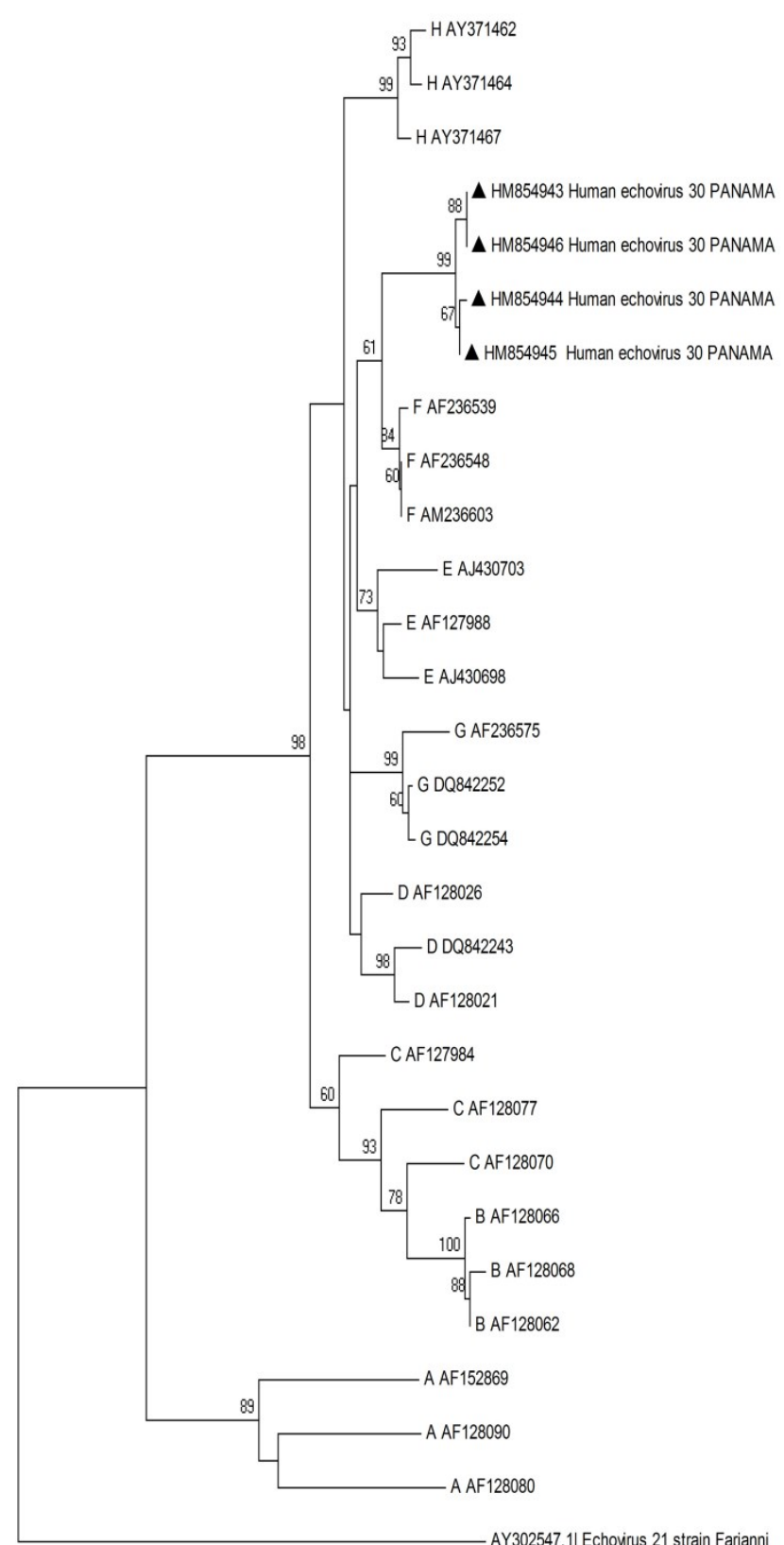

0.08

Phylogenetic tree showing the relationship between the Panamanian samples (bold circle) and Echovirus 30 strains available in GenBank. The tree is rooted with Echovirus 21 Farianni strain. Both trees were constructed using the Neighborjoining method with Bootstrap 1000 replicates and the maximum composite likelihood method was used to evaluate genetic distances in the MEGAv4.1 software. 
absence of neck signs, however, meningitis should not be excluded from diagnosis.

Our main goal was a molecular comparison of the Echovirus 30 sequences obtained from Panama with those from other outbreaks. However, no sequence data was reported in the Japanese publication; the echovirus 30 sequences from Korea and Argentina were from the H group and the Panamanian sequences were from the $\mathrm{F}$ group. A phylogenetic analysis of Panamanian and Korean samples demonstrated that they clustered in different lineages (data not shown) and a comparison with the echovirus 30 sequences from Argentina was not possible; different parts of the virus genome had been sequenced.

To our best knowledge, this is the first defined outbreak of enterovirus and aseptic meningitis in Panama, and Central America more broadly, since the early 1980 s. In that decade, acute fevers and aseptic meningitis were reported in epidemic proportions, affecting 1,023 patients, the majority of whom were less than 15 years of age and resident in Panama City [14]. The causative agent was an enterovirus identified by serological methods as echovirus 4, another member of the human enterovirus B species [14]. In 1981, an outbreak of acute hemorrhagic conjunctivitis was observed in the Colon Province of Panama, with the responsible agent identified as enterovirus 70, a member of the human enterovirus D species [15]. In 2002, another outbreak of aseptic meningitis similar to that described in the present report occurred in Chiriquí Province, but the causative agent of the meningitis was not defined. In this previous outbreak, 261 cases were reported between May and July, compared to the 173 cases observed between May and June of 2008 (Pablo Acosta, personal communication, September 2009). This time of year is significant in Panama for the onset of the rainy season and increasing humidity, and we hypothesize that climatic conditions may have encouraged people to stay indoors, facilitating person-to-person spread of the pathogen.

In the past 40 years, echoviruses have been an important recurring cause of large outbreaks of aseptic meningitis throughout the world $[6,9,16]$, alone or in combination with other enteroviruses [3]. Echovirus 30 undergoes several years of quiescence between outbreaks $[6,12]$, indicating that public health services must anticipate and be prepared to identify and manage outbreaks about once every 10 to 15 years. The molecular epidemiological study presented here demonstrates the presence of an enterovirus in Panama that can act as a causative agent of aseptic meningitis.
The cases of echovirus 30 in Chiriqui Province demonstrate the need for Panama to develop and maintain the appropriate infrastructure to conduct timely diagnosis in situations of severe and widespread outbreaks of aseptic meningitis.

\section{Conclusion}

We have detected an aseptic meningitis outbreak in children where the likely agent was enterovirus type viruses in which echovirus 30 was identified in a group of samples; this is the first reported outbreak of enterovirus in Panama, and possibly Central America, since the 1980s.

\section{Acknowledgements}

Thanks go to the Gorgas Memorial Institute for Health Studies for funds to support this project and to the members of the Department of Epidemiology, Ministry of Health, David, Chiriquí Province, for their work in providing the epidemiological information for this project. We thank the two anonymous reviewers for their many helpful suggestions for improving the manuscript.

\section{References}

1. Mirand A, Henquell C, Archimbaud C, Peigue-Lafeuille H, Bailly JL (2007) Emergence of recent echovirus 30 lineages is marked by serial genetic recombination events. J Gen Virol 88: 166-176.

2. Hyypiä T (2008) Echoviruses. In: Mahy BWJ, van Regenmortel MHV, editors. Encyclopedia of Virology: Amsterdam: Elsevier p. 68-71.

3. Begier EM, Oberste MS, Landry ML, Brennan T, Mlynarski D, Mshar PA, Frenette K, Rabatsky-Ehr T, Purviance K, Nepaul A, Nix WA, Pallansch MA, Ferguson D, Cartter ML, Hadler JL (2008) An outbreak of concurrent echovirus 30 and coxsackievirus A1 infections associated with sea swimming among a group of travelers to Mexico. Clin Infect Dis 47: 616-623.

4. Hayashi T, Shirayoshi T, Nagano T, Yaoita H, Kogure S, Nariai H, Natsumeda T, Taniuchi M, Sandoh M, Sato Y (2009) An outbreak of aseptic meningitis due to echovirus 30 in a high school baseball club--possible role of severe exercise for a high attack rate. Intern Med 48: 1767-1771.

5. Dos Santos GP, Skraba I, Oliveira D, Lima AA, de Melo MM, Kmetzsch CI, da Costa EV, da Silva EE (2006) Enterovirus meningitis in Brazil, 1998-2003. J Med Virol 78: 98-104.

6. Oberste MS, Maher K, Kennett ML, Campbell JJ, Carpenter MS, Schnurr D, Pallansch MA (1999) Molecular epidemiology and genetic diversity of echovirus type 30 (E30): genotypes correlate with temporal dynamics of E30 isolation. J Clin Microbiol 37(: 3928-3933.

7. Farias A, Cabrerizo M, Re V, Glatstein N, Pisano B, Spinsanti L, Contigiani MS (2011) Molecular identification of human enteroviruses in children with neurological infections from the central region of Argentina. Arch Virol 156: 129-133.

8. Choi YJ, Park KS, Baek KA, Jung EH, Nam HS, Kim YB, Park JS (2008) Molecular characterization of echovirus 30- 
associated outbreak of aseptic meningitis in Korea in 2008. J Microbiol Biotechnol 20: 643-649.

9. Zhao YN, Jiang QW, Jiang RJ, Chen L, Perlin DS (2005) Echovirus 30, Jiangsu Province, China. Emerg Infect Dis 11: 562-567.

10. Iturriza-Gomara M, Megson B, Gray J (2006) Molecular detection and characterization of human enteroviruses directly from clinical samples using RT-PCR and DNA sequencing. $J$ Med Virol 78: 243-253.

11. Bailly JL, Mirand A, Henquell C, Archimbaud C, Chambon M, Charbonne F, Traoré O, Peigue-Lafeuille H (2009) Phylogeography of circulating populations of human echovirus 30 over 50 years: nucleotide polymorphism and signature of purifying selection in the VP1 capsid protein gene. Infect Genet Evol 9: 699-708.

12. Khetsuriani N, Lamonte-Fowlkes A, Oberst S, Pallansch MA (2006) Enterovirus surveillance--United States, 1970-2005. MMWR Surveill Summ 55: 1-20.

13. Michos AG, Syriopoulou VP, Hadjichristodoulou C, Daikos GL, Lagona E, Douridas P, Mostrou G, Theodoridou M (2007) Aseptic meningitis in children: analysis of 506 cases. PLoS One 2: e674.

14. Reeves WC, Quiroz E, Brenes MM, Centeno R, Campos G (1987) Aseptic meningitis due to echovirus 4 in Panama City, Republic of Panama. Am J Epidemiol 125: 562-575.
15. Reeves WC, Brenes MM, Quiroz E, Palacios J, Campos G, Centeno R (1986) Acute hemorrhagic conjunctivitis epidemic in Colon, Republic of Panama. Am J Epidemiol 123: 325335 .

16. Outbreaks of aseptic meningitis associated with echoviruses 9 and 30 and preliminary surveillance reports on enterovirus activity--United States, 2003. MMWR Morb Mortal Wkly Rep. 2003 52: 761-764.

\section{Corresponding author}

Juan M. Pascale

Apartado Postal 0816 - 02593

Instituto Conmemorativo de Estudios de la Salud

Avenida Justo Arosemena y Calle 35

Bella Vista, Panamá, Republica de Panamá

Telephone: (507) 527-4811

Fax: (507) 527-4889

Email address: jpascale@gorgas.gob.pa

Conflict of interests: No conflict of interests is declared. 